
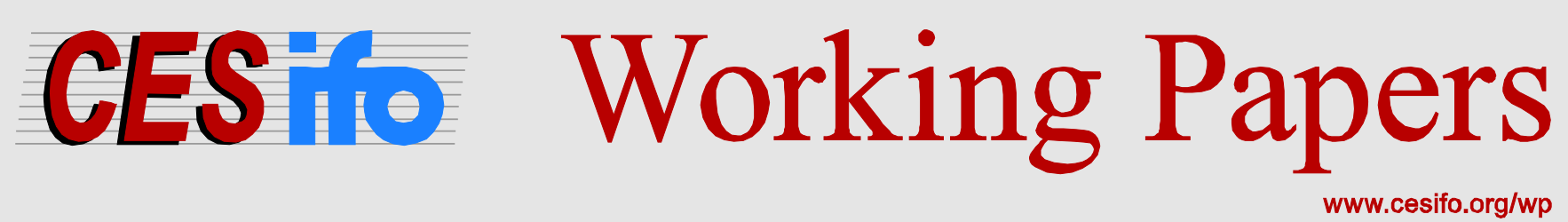

\title{
Distribution-neutral Welfare Ranking-Extending Pareto Principle
}

\author{
Sugata Marjit \\ Sandip Sarkar
}

\section{CESIFO WORKING PAPER NO. 6397 \\ CATEgORY 1: PUBlic FinANCE \\ MARCH 2017}
An electronic version of the paper may be downloaded
- from the SSRN website:
- from the RePEc website:
- from the CESifo website:
www.SSRN.com
www.RePEc.org
www.CESifo-group.org/wp




\title{
Distribution-neutral Welfare Ranking-Extending Pareto Principle
}

\begin{abstract}
The well known Pareto criterion used in the context of efficiency and welfare has to do with absolute changes whereas in every domain of economic behaviour inequality or relative changes has become a major concern. We propose an inequality-preserving or distribution neutral Pareto criterion-the strong Pareto superior or SPS allocation which preserves the initial distribution and makes everyone better off. Our main result is that whenever there is a gain in the aggregate value of the relevant attribute, there exists a unique counterfactual allocation which is SPS.
\end{abstract}

JEL-Codes: A100.

Keywords: Pareto superiority, strong Pareto superiority, inequality.

Sugata Marjit

Centre for Studies in Social Sciences

Calcutta / India

marjit@gmail.com
Sandip Sarkar

Centre for Studies in Social Sciences

Calcutta / India

sandip.isi.08@gmail.com

We sincerely acknowledge Sugata Bhattacharya, B. Ravikumar, S.M. Ravi Kanvur, Sanjeev Gupta, Saibal Kar, Pranab Kumar Das and Arindam Paul for helpful comments in an earlier version of this paper. 


\section{Introduction}

Pareto ranking or Pareto efficiency is a topic economists are exposed to very early in their career. In particular the basic welfare comparison between two social situations starts with the ranking in terms of a principle Pareto have talked about in the nineteenth century. If we compare two social situations $A$ and $B$, we say $B$ is Pareto superior to $A$ iff everyone is as well of in $B$ as in $A$ and at least one is strictly better off in situation $B$ compared to $A$. This comparison is done in terms of utility or welfare levels individuals enjoy in $A$ and $B$. In the theory of social welfare this has been a widely discussed topic with seminal contributions from De Scitovszky (1941), Samuelson (1958), Arrow (1963), Stiglitz (1987), Sen (1970) and others to make recent treatments such as Mandler (1999), Cornes and Sandler (2000) and many others.

Pareto's principle provides a nice way to compare situations when some gain and some lose by considering whether transfer from gainers to losers can lead to a new distribution in $B$ such that $B$ turns out to be Pareto superior to $A$, the initial welfare distribution. It is obvious that if sum of utilities increases in $B$ relative to $A$, then whatever be the actual distribution in $B$, a transfer mechanism will always exist such that transfer-induced redistribution will make $B$ Pareto superior to $A$. The great example is how gains from international trade can be redistributed in favor of those who lose from trade such that everyone gains due to trade. Overall gains from trade lead to a highly level of welfare, under ideal conditions and therefore one can show that under free trade eventually nobody may lose as gainers 'bribe' the losers. But whatever it is Pareto ranking definitely does not address the inequality issue. There will be situations where $B$ will be Pareto superior to $A$, but inequality in $B$ can be much greater than $A$. The purpose of this paper is to extend the basic principle of Pareto's welfare ranking subjecting it to a 
stricter condition that keeps the degree of inequality intact between $A$ and $B$ after transfer from gainers to losers, but at the same time guaranteeing that everyone gains in the end.

Thus we coin a Strong-Pareto criterion which not only insists that everyone must be better of in $B$ compared to $A$, but also requires that degree of inequality must remain the same between $A$ and $B$. Only then $B$ will be Strongly Pareto Superior (SPS) to A. Concern for such a Strong principle stems from the fact that people do care about inequality and inequality has become a worldwide popular point of debate in public domain (see Stewart, 2004; Stiglitz, 2012; Piketty, 2014; Atkinson and Stiglitz, 2015, for further readings).

Pareto superior move as such may not contain agitation to change policies further because of rising inequality. We are also motivated by the query as to whether the basic condition that guarantees Pareto superiority of $B$ to $A$, would also guarantee that $B$ is SPS to $A$. Apparently it need not be since there can be transfer that make $B$ PS $A$, but that aggravate inequality.

We show that if total utility in $B$ is greater than that of $A$, we can always construct a counterfactual distribution $C$ which is SPS to $A$. We refer $C$ as a SPS allocation. The construction of SPS depends on whether absolute or relative inequality measures are preserved. In order to keep the relative inequality level same we redistribute the aggregate gains from $B$ to $A$ proportionate to that of individuals utility as in $A$. On the other hand we preserve the absolute inequality by equally distributing the aggregate gains to the individual utilities of $A$. We show that the SPS allocations generated by this mechanism is Pareto superior to any other allocation which also preserves either relative or absolute inequality. We also show that the SPS allocation that preserves absolute inequality can be obtained from the relative SPS 
allocation and also from the final distribution by sequence of mean preserving transfer.

The rest of the paper is organized as follows. The next section starts with an introduction to the notations, assumptions and definitions used throughout the paper. Section 3 contains the main results associated with SPS. Last section concludes.

\section{Preliminaries}

Consider an $n(n>1)$ agent society whose utility profiles being observed for two time points. The initial time point is denoted by 0 , whereas the final time point is denoted by 1 . Let the utility profile at the initial period is given by $U_{0}=\left\{u_{1}, u_{2}, \ldots, u_{n}\right\}$, where $u_{i}$ denotes utility of the $i^{t h}$ individual. We make the following assumptions on $u_{i}$ :

Assumption 1. $u_{i}$ is cardinally measurable $\forall i \in\{1,2, . ., n\}$.

Assumption 2. $u_{i}>0 \forall i \in\{1,2, . ., n\}$.

Let $\mathbb{D}^{n}$ be the set of all utility profiles with number of agents being $n$ where utility of each individual satisfies assumptions 1 and 2. Consider $U_{1}=\left\{g_{1} u_{1}, g_{2} u_{2}, \ldots, g_{n} u_{n}\right\}$ be the utility profile at time 1 , where utility of individual $i$ grows by $g_{i}$, such that:

Assumption 3. $g_{i}>0 \forall i \in\{1,2, . ., n\}$.

Given assumption 3 we can write $U_{1} \in \mathbb{D}^{n}$.

In the theoretical foundations of this paper we often use the notions of relative and absolute inequality measures. We define these in the following fashion: 
Definition 1. Relative inequality indices: Any inequality index I : $\mathbb{D}^{n} \longrightarrow \mathbb{R}$ is said to belong in the family of relative inequality indices if and only if the inequality index is homogeneous of degree zero, i.e., $I\left(u_{1}, u_{2}, . ., u_{n}\right)=$ $I\left(\delta u_{1}, \delta u_{2}, . ., \delta u_{n}\right) ; \forall \delta \in \mathbb{R}$ and $\delta>0$.

Definition 2. Absolute inequality indices: Any inequality index I : $\mathbb{D}^{n} \longrightarrow \mathbb{R}$ is said to belong in the family of absolute inequality indices if and only if the inequality index is translation invariant, i.e., $I\left(u_{1}, u_{2}, . ., u_{n}\right)=$ $I\left(\theta+u_{1}, \theta+u_{2}, . ., \theta+u_{n}\right) ; \forall \theta \in \mathbb{R}$ and $\theta>0$.

Most of the popular inequality indices like Gini coefficient, Theils measure, Atkinson's family of inequality indices belongs in the class of relative inequality measures. Variance is a popular absolute inequality measure. There are pros and cons of using both these measures. The advantage of using relative measures is that inequality of a society does not depend on the choice of unit of analysis (e.g., Pound Sterlings, US dollar, Indian rupees etc). Unlike relative measures, absolute inequality measures depends on the choice of unit. In order to illustrate the rationality of using the absolute inequality measures consider $X=\{1,10\}$ and $Y=\{10,100\}$ as the initial and the final utility profiles, respectively. The absolute gain of the richer is 90 and that of the poorer is 9 . However, although rich becomes richer in comparison to the poor the degree of relative inequality in $Y$ is same as $X$. Absolute inequality measures are free from such criticisms. Kolm (1976a,b) referred the class of absolute inequality measures as the leftist inequality measures.

\section{Strong Pareto Superiority}

Pareto superiority (PS) is defined as the situation where no one looses from the initial to the final period but at least one individual gains. However, 
PS allocation may aggravate inequality. We thus introduce "Strong Pareto Superiority" (SPS). By SPS we mean a situation where the utility of all the individuals increases and the inequality (either absolute or relative) also remains same, compared to that of the initial distribution. In order to derive such a SPS allocation we first assume that their exists a social planner who taxes a subgroup of the population and distributes the collected tax to the rest of the population. Note that as we move through this paper we show that in general the SPS allocations and their associated tax transfer vector will be different for the relative and absolute inequality measures. From here onwards we refer the SPS allocation's that preserves relative and absolute inequality as relative-SPS and absolute-SPS, respectively.

Before we move through the main contributions of this paper we begin with a brief illustration on SPS allocations restricting the number of agents to 2 . For this we assume that there exists a $T \in \mathbb{R}$ such that:

$$
\frac{u_{1}}{u_{2}}=\frac{g_{1} u_{1}-T}{g_{2} u_{2}+T}
$$

We can solve $T$ from the above equation in the following fashion

$$
T=\frac{u_{1} u_{2}\left(u_{1}-u_{2}\right)}{g_{1} u_{1}+g_{2} u_{2}}
$$

Note that the utility profiles $u=\left\{u_{1}, u_{2}\right\}$ and $\hat{u}=\left\{\hat{u}_{1}=g_{1} u_{1}-T, \hat{u}_{2}=\right.$ $\left.g_{2} u_{2}+T\right\}$ have same level of inequality, following any measure that is relative in nature. Further, $\hat{u}_{1}>u_{1}$ and $\hat{u}_{2}>u_{2}$, holds if the society enjoys positive growth (i.e., $g_{1} u_{1}+g_{2} u_{2}>u_{1}+u_{2}$ ). Hence $\hat{u}=\left\{\hat{u}_{1}, \hat{u}_{2}\right\}$ qualifies as a relative-SPS allocation. Similarly we can design the absolute-SPS allocation, where $T$ has to be solved from the following absolute inequality preserving condition: $u_{1}-u_{2}=\left(g_{1} u_{1}-T\right)-\left(g_{2} u_{2}-T\right)$ 
Before we generalize this result to the case $n>2$, we formally define SPS as follows:

Definition 3. SPS allocation For all $U_{0}, U_{1} \in \mathbb{D}^{n}$, any allocation $\hat{U}=$ $\left\{\hat{u}_{1}, \hat{u}_{2}, . ., \hat{u}_{n}\right\} \in \mathbb{D}^{n}$ is said to be a relative-SPS allocation to $U_{0}$ which is denoted by $\hat{U} \succ_{R S P S} U_{0}$, if and only if $\hat{u}_{i}>u_{i}$ and $\frac{u_{i}}{u_{j}}=\frac{\hat{u}_{i}}{\hat{u}_{j}}, \forall i, j \in\{1,2, . ., n\}$. Any allocation $\hat{V}=\left\{\hat{v}_{1}, \hat{v}_{2}, . ., \hat{v}_{n}\right\} \in \mathbb{D}^{n}$ is said to be an absolute-SPS allocation to $U_{0}$ which is denoted by $\hat{V} \succ_{A S P S} U_{0}$, if and only if $\hat{v}_{i}>u_{i}$ and $u_{i}-u_{j}=\hat{v}_{i}-\hat{v}_{j}, \forall i, j \in\{1,2, . ., n\}$.

Note that if we scale up utilities of all the individual's of the initial allocation by any positive scalar greater than 1 , we necessarily get a relative-SPS allocation. Nevertheless, such an allocation is not feasible if the aggregate utility of the counterfactual allocation exceeds that of the final allocation. We define this formally as follows:

Definition 4. Feasible SPS allocation: For all $U_{0}, U_{1} \in \mathbb{D}^{n}$, and any $\hat{U}=\left\{\hat{u}_{1}, \hat{u}_{2}, . ., \hat{u}_{n}\right\} \in \mathbb{D}^{n}$ is said to be a feasible relative-SPS or a feasible absolute-SPS allocation if $\sum_{i=1}^{n} \hat{u}_{i} \leq \sum_{i=1}^{n} u_{1 i}$.

It is possible that for any feasible SPS allocation, some resource may remain as a residual which can be further redistributed amongst the agents to make every one better off (for example if $\sum_{i=1}^{n} \hat{u}_{i}<\sum_{i=1}^{n} u_{1 i}$ ). From the set of all feasible SPS allocations we refer a SPS allocation to be the best (BSPS) if and only if it is Pareto superior to any other feasible SPS allocation. In general BSPS will not be the same for the relative and absolute inequality measures. However, they are related. We explore this towards the end of this section. Next we define BSPS formally:

Definition 5. Best SPS allocation: For all $U_{0}, U_{1} \in \mathbb{D}^{n}$, any allocation $\hat{U}=\left\{\hat{u}_{1}, \hat{u}_{2}, . ., \hat{u}_{n}\right\} \in \mathbb{D}^{n}$, is said to be the best relative-SPS allocation denoted 
by $\hat{U} \succ_{B R S P S} U_{0}$ if and only if $\hat{U}$ is Pareto Superior to any other feasible relative-SPS allocation. Any allocation $\hat{V}=\left\{\hat{v}_{1}, \hat{v}_{2}, . ., \hat{v}_{n}\right\} \in \mathbb{D}^{n}$ is said to be the best absolute-SPS allocation denoted by $\hat{V} \succ_{B A S P S} U_{0}$ if and only if $\hat{V}$ is Pareto Superior to any other feasible absolute-SPS allocation.

We now characterize one important property associated with the relative and absolute BSPS allocation.

Remarks 1. For all $U_{0}, U_{1} \in \mathbb{D}^{n}$ and for any $\hat{U}=\left\{\hat{u}_{1}, \hat{u}_{2}, . ., \hat{u}_{n}\right\} \in \mathbb{D}^{n}$ the following condition holds

1) $\hat{U} \succ_{B R S P S} U_{0} \Longrightarrow \sum_{i=1}^{n} \hat{u}_{i}=\sum_{i=1}^{n} g_{i} \cdot u_{i}$

2) $\hat{U} \succ_{B A S P S} U_{0} \Longrightarrow \sum_{i=1}^{n} \hat{u}_{i}=\sum_{i=1}^{n} g_{i} \cdot u_{i}$.

The proof is straightforward and is thus omitted.

Given remarks 1 we now characterize the relative and absolute BSPS allocations in the following proposition:

Proposition 1. For all $U_{0}, U_{1} \in \mathbb{D}^{n}$, and for any $\hat{U}=\left\{\hat{u}_{1}, \hat{u}_{2}, . ., \hat{u}_{n}\right\} \in \mathbb{D}^{n}$ the following conditions hold:

1) $\hat{U} \succ_{B R S P S} U_{0} \Longleftrightarrow \sum_{i=1}^{n} g_{i} u_{i}>\sum_{i=1}^{n} u_{i}$ and $\hat{u}_{i}=u_{i}\left(\frac{\sum_{i=1}^{n} g_{i} u_{i}}{\sum_{i=1}^{n} u_{i}}\right), \forall i \in$ $\{1,2, \ldots, n\}$

2) $\hat{U} \succ_{B A S P S} U_{0} \Longleftrightarrow \sum_{i=1}^{n} g_{i} u_{i}>\sum_{i=1}^{n} u_{i}$ and $\hat{u}_{i}=u_{i}+\left(\frac{\sum_{i=1}^{n} g_{i} u_{i}-\sum_{i=1}^{n} u_{i}}{n}\right), \forall i \in$ $\{1,2, \ldots, n\}$.

Proof: We provide the proof only for statement 1 . Statement 2 can be proved using same logic.

Only if

Given $\hat{U} \succ_{B R S P S} U_{0} \Longrightarrow \exists \theta \in \mathbb{R}$ such that $\hat{u}_{i}=\theta . u_{i} \forall i \in\{1,2, . ., n\}$ and $\theta>1$. Now since $\hat{U}$ is a BSPS, following Remarks 1 we can write 
$\sum_{i=1}^{n} \hat{u}_{i}=\sum_{i=1}^{n} g_{i} u_{i} \Longrightarrow$

$$
\theta=\frac{\sum_{i=1}^{n} g_{i} u_{i}}{\sum_{i=1}^{n} u_{i}}
$$

Since $\hat{u}_{i}=\theta u_{i}$ putting the value of $\theta$ we get the desiderata. Further, $\theta>1 \Longrightarrow \sum_{i=1}^{n} g_{i} u_{i}>\sum_{i=1}^{n} u_{i}$.

If

Given $\hat{u}_{i}=u_{i} \cdot\left(\frac{\sum_{i=1}^{n} g_{i} u_{i}}{\sum_{i=1}^{n} u_{i}}\right)$ and $\sum_{i=1}^{n} g_{i} u_{i}>\sum_{i=1}^{n} u_{i} \quad \Longrightarrow \quad \hat{u}_{i}>u_{i}$. Clearly $\hat{U}$ and $U_{0}$ have same inequality, following any the class of relative inequality measures defined in 1 . Furthermore, it is readily observable that $\sum_{i=1}^{n} \hat{u}_{i}=\sum_{i=1}^{n} g_{i} u_{i}$. Combining these three arguments it is straightforward to write that $\hat{U} \succ_{B R S P S} U_{0}$. Q.E.D.

Following the previous discussions it is clear that in general it is necessary to tax a set of individuals and redistribute the collected tax to the rest of the individuals for obtaining the SPS allocations (relative and absolute). The tax transfer vector for the relative best SPS allocations is given by

$$
T^{R}=\left\{T_{1}^{R}, T_{2}^{R}, \ldots, T_{n}^{R}\right\}
$$

where $T_{i}^{R}=u_{i}\left(g_{i}-\frac{\sum_{i=1}^{n} g_{i} u_{i}}{\sum_{i=1}^{n} u_{i}}\right)$.

On the other hand tax transfer vector associated with the absolute best SPS allocations is given by:

$$
T^{A}=\left\{T_{1}^{A}, T_{2}^{A}, \ldots, T_{n}^{A}\right\}
$$

where $T_{i}^{A}=u_{i}\left(g_{i}-1\right)-\left(\frac{\sum_{i=1}^{n} g_{i} u_{i}-\sum_{i=1}^{n} u_{i}}{n}\right)$.

It is clear that an individual has to pay tax (receives transfer) following the relative and the absolute-BSPS allocations if and only if $T_{i}^{R}>0\left(T_{i}^{R}<0\right)$ and $T_{i}^{A}>0\left(T_{i}^{A}<0\right)$, respectively.

In the next result we show that the tax transfer vectors $T^{R}$ and $T^{A}$ are 
unique.

Proposition 2. The tax transfer vectors $T^{R}$ and $T^{A}$ (defined in equations 4 and 5) associated with relative and absolute BSPS allocation, respectively are unique.

Proof: We provide the proof for uniqueness only for $T^{R}$. However, uniqueness of $T^{A}$ can be proved using the same logic.

Let $\hat{U}=\left\{\hat{u}_{1}, \hat{u}_{2}, . ., \hat{u}_{n}\right\} \succ_{B R S P S} U_{0}$. We begin with the assumption that there exists any arbitrary $\bar{T}=\left\{\bar{T}_{1}, \bar{T}_{2}, . ., \bar{T}_{n}\right\} \in \mathbb{R}^{n}$ associated with any SPS allocation $\bar{U}=\left\{\bar{u}_{1}, \bar{u}_{2}, . ., \bar{u}_{n}\right\}$ such that $\bar{T} \neq T^{R}$. In order to complete the proof we show that if $\bar{U}$ qualifies as a BRSPS then $\bar{U} \equiv \hat{U}$ and $\bar{T} \equiv T^{R}$. Note that any two vectors of the same order are related in the following fashion: $\bar{T}=T+\epsilon$ where $\epsilon=\left\{\epsilon_{1}, \epsilon_{2}, . ., \epsilon_{n}\right\} \in \mathbb{R}^{n}$. Since $T \neq \bar{T} \Longrightarrow \exists i \in\{1,2, . ., n\}$ such that $\epsilon_{i} \neq 0$. Clearly, inequality in $\bar{U}$ is same as $U_{0}$ following definition 1 if and only if $\exists \alpha(\alpha \in \mathbb{R})$ such that $\epsilon_{i}=\alpha u_{i}, \forall i \in\{1,2, . ., n\}$. Hence we can write $\bar{u}_{i}=u_{i} \cdot(\theta-\alpha)$, where $\theta=\frac{\sum_{i=1}^{n} g_{i} u_{i}}{\sum_{i=1}^{n} u_{i}}$. Following remarks 1 if $\alpha \neq 0$ then $\bar{U}$ does not qualifies as a best SPS allocation. Hence, $\alpha=0 \Longrightarrow \bar{U} \equiv \hat{U}$ and $\bar{T} \equiv T^{R}$. Q.E.D.

We are ready to establish a relationship between the absolute and the relative BSPS allocations. For this we consider the idea of mean preserving spread a well known terminology in the income inequality literature. Loosely speaking it refers transfer of income from two persons such that the recipient is not becoming rich as a result of the transfer. Formally we define this as follows:

Definition 6. Mean Preserving Transfer (MPT): For any $X=\left\{X_{1}, X_{2}\right.$ ,..,$\left.X_{n}\right\}, Y=\left\{Y_{1}, Y_{2}, . ., Y_{n}\right\}$, such that $X_{l+1} \geq X_{l} \geq 0$ and $Y_{l+1} \geq Y_{l} \geq 0$. We say that $X$ is said to be obtained from $Y$ by a mean preserving trans- 
fer such that for any $i, j, k \in\{1,2, . ., n\}, i>j$ and $k \neq i, j$ we have $X_{i}=$ $Y_{i}-\epsilon, X_{j}=Y_{j}+\epsilon, X_{k}=Y_{k}$.

We now show that the absolute BSPS allocation can be obtained from the relative BSPS allocation and also from the final distribution following a sequence of mean preserving transfer defined in 6 .

Proposition 3. For all $U_{0}, U_{1} \in \mathbb{D}^{n}$, and let there exists $\hat{U}=\left\{\hat{u}_{1}, \hat{u}_{2}, . ., \hat{u}_{n}\right\} \in$ $\mathbb{D}^{n}$ and $\hat{V}=\left\{\hat{v}_{1}, \hat{v}_{2}, . ., \hat{v}_{n}\right\} \in \mathbb{D}^{n}$ such that $\hat{U} \succ_{B R S P S} U_{0}$ and $\hat{V} \succ_{B A S P S} U_{0}$. Then $\hat{V}$ is obtained following finite sequences of mean preserving transfers from $U_{1}$ and also from $\hat{U}$.

Proof: In order to prove this proposition we use a very well known result introduced in a seminal article by Atkinson (1970). This result states that any profile $X=\left\{X_{1}, X_{2}, . ., X_{n}\right\}$ is obtained form other another profile $Y=\left\{Y_{1}, Y_{2}, . ., Y_{n}\right\}$ (where $X_{i} \geq 0$ and $Y_{i} \geq 0 \forall i \in\{1,2, . ., n\}$ ) following a sequence MPT if and only if both the following conditions are satisfied: 1) Lorenz curve of $X$ lies above $Y$ at least in one point and not below $Y$ in any point, which we denote as $X \succ_{\text {Lorenz }} Y$. 2) $\sum_{i=1}^{n} X_{i}=\sum_{i=1}^{n} Y_{i}$.

In the present context it is quite straightforward to show that $\hat{V} \succ_{\text {Lorenz }} \hat{U}$ and $\hat{V} \succ_{\text {Lorenz }} U_{1}$. Furthermore, following remarks 1 we have $\sum_{i=1}^{n} \hat{v}_{i}=$ $\sum_{i=1}^{n} \hat{u}_{i}=\sum_{i=1}^{n} g_{i} u_{i}$. Since population size is constant we can use Atkinson's theorem mentioned in the previous paragraph in order to complete the proof.

\section{Q.E.D.}

An inequality index which does not increase as a result of MPT is said to satisfy transfer axiom. Most of the widely used indices like Gini, Theil, satisfies this axiom. The proposition stated above also implies that any inequality index which satisfies transfer axiom will never exhibit higher inequality in the absolute BSPS profile compared to that of the relative BSPS allocation and the final utility profile. 


\section{Conclusion}

We have extended the basic Pareto principle to focus on inequality-neutral or distribution neutral Pareto superior allocation which we call strongly Pareto superior or SPS allocation which guarantees higher individual welfare keeping the degree of inequality same as before. We have shown that whenever the society experiences aggregate gain we can compute the SPS allocation by taxing a subgroup of population and redistributing the collected tax to the rest of the population. The construction of SPS is different when relative and absolute inequality is preserved. The SPS allocation preserving the relative inequality is obtained by redistribution of the aggregate gains among the individuals proportional to their utilities of the initial distribution. On the other hand the SPS allocation which preserves absolute inequality is obtained by equally distributing the aggregate gains among all the individuals. We also show that the SPS allocation that preserves absolute inequality can be obtained from the relative SPS allocation and also the final distribution by sequence of mean preserving transfer.

Our approach retains the spirit of Pareto criterion but instead of keeping the absolute level intact, we keep the inequality level the same. Any Pareto superior allocation can be converted to a SPS allocation.

\section{References}

Arrow, K. J. (1963). Social Choice and Individual Values. Number 12. Yale University Press.

Atkinson, A. (1970). On the measurement of inequality. Journal of Economic Theory, 2:244-263. 
Atkinson, A. B. and Stiglitz, J. E. (2015). Lectures on public economics. Princeton University Press.

Cornes, R. and Sandler, T. (2000). Pareto-improving redistribution and pure public goods. German Economic Review, 1(2):169-186.

De Scitovszky, T. (1941). A note on welfare propositions in economics. The Review of Economic Studies, 9(1):77-88.

Kolm, S.-C. (1976a). Unequal inequalities. i. Journal of Economic Theory, 12(3):416-442.

Kolm, S.-C. (1976b). Unequal inequalities. ii. Journal of Economic Theory, $13(1): 82-111$.

Mandler, M. (1999). Simple pareto-improving policies. Journal of economic theory, 84(1):120-133.

Piketty, T. (2014). Capital in the twenty-first century. Harvard University Press.

Samuelson, P. A. (1958). Aspects of public expenditure theories. The Review of Economics and Statistics, pages 332-338.

Sen, A. (1970). Collective choice and social welfare.

Stewart, F. (2004). Development and security. Conflict, Security $\&$ Development, 4(3):261-288.

Stiglitz, J. (2012). The price of inequality. Penguin UK.

Stiglitz, J. E. (1987). Pareto efficient and optimal taxation and the new new welfare economics. Handbook of public economics, 2:991-1042. 